\title{
Function Decomposition and Synthesis Using Linear Sifting
}

\author{
Christoph Meinel \\ FB IV - Informatik \\ Univ. Trier \\ D - 54286 Trier
}

\author{
Fabio Somenzi \\ Electr. and Computer Engineering \\ Univ. of Colorado at Boulder \\ USA - Boulder, CO 80309-0425
}

\author{
Thorsten Theobald \\ Zentrum Mathematik \\ TU München \\ D - 80290 München
}

\begin{abstract}
In order to simplify a synthesis task for particularly hard functions it is sometimes inevitable to decompose the function in a preprocessing step. We propose a new algorithm for automatically decomposing a target function by extracting a linear filter within the synthesis process. The algorithm is an application of the Linear Sifting algorithm which has been proposed in [6]. Using this method we were able to synthesize functions with standard tools which fail otherwise.
\end{abstract}

\section{Introduction}

Decomposition techniques date back to the very early days of computer-aided circuit design [5]. By revealing the structural properties of a switching function it is possible to establish representations by means of several simpler functions. Utilizing these functional properties enables us to decompose large and complex circuits into a system of smaller subcircuits which may be readily available and economically maintained.

Recent developments concerning symbolic representations of switching functions by means of decision diagrams [2] have renewed the interest in decomposition techniques $[3,11]$ : By extracting functional properties and decomposing the switching functions the well-known power of symbolic manipulation techniques can be even further extended.

In particular, the recent work [3] dealt with the problem that most multi-level synthesis tools like SIS run into severe problems for complex functions. By decomposing the circuit into the cascade of two suitable subcircuits the synthesis task can be simplified. However, efficient algorithms to find such a decomposition are available only for very specific types of decomposition. An attractive candidate is to divide the function $f$ into a linear block $\sigma$ and a non-linear block $f^{\prime}$ (see Fig. 1, [15]) such that $f(x)=f^{\prime}(\sigma(x))$. The main synthesis task now is to find a linear block $\sigma$ that minimizes the complexity of $f$. In [3] this problem has been tackled by methods of spectral analysis. However, although there is a well-established theory on these techniques [4] the practical implementation of these ideas leads to very complex algorithms and the improvements are limited. Hence, an important question is not only to find better algorithms but also to find algorithms which can be integrated much more easily into existing design systems.

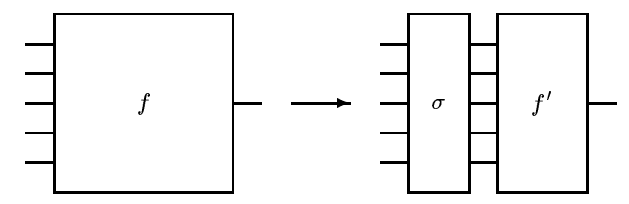

Figure 1: Linear decomposition.

${ }^{*}$ This work was supported in part by NSF grant MIP-94-22268, SRC contract 95-DJ-560 and DFG-grant Me 1077/12-1.
In this paper we propose to use the Linear Sifting algorithm [6] for the computation of the linear block $\sigma$. This algorithm is an extension of the well-known Sifting algorithm [12], which is currently the state-of-the-art method for finding good variable orderings of binary decision diagrams. In the Linear Sifting algorithm, not only swaps of two neighboring variables are applied as elementary operations, but also linear transformations among neighboring variables. This algorithm modifies a given function, but preserves the canonicity in representation and the efficiency of manipulation. We show in what respect Linear Sifting can be used to perform the above mentioned synthesis task, and we prove the validity of our approach by experimental results.

The paper is structured as follows: In Section 2 we recall definitions relevant to decision diagrams and linear transformations. In Section 3 we review the approach of [3] based on spectral analysis. In Section 4 we present our new approach based on Linear Sifting and contrast it to the existing approach. Experimental results are given in Section 5. Finally, the ideas and results are summarized.

\section{Preliminaries}

\subsection{Binary Decision Diagrams}

Ordered binary decision diagrams (OBDDs) [2] are rooted directed acyclic graphs representing switching functions. Each OBDD has two sink nodes which are labeled 1 and 0 . Each internal ( $=$ non-sink) node is labeled by an input variable $x_{i}$ and has two outgoing edges, labeled 1 and 0 . A linear variable order $\pi$ is placed on the input variables. The variable occurrences on each OBDD-path have to be consistent with this order. An OBDD computes a switching function $f: \mathbb{B}^{n} \rightarrow \mathbb{B}$, $\mathbb{B}=\{0,1\}$, in a natural manner: each assignment to the input variables $x_{i}$ defines a unique path through the graph from the root to a sink. The label of this sink defines the value of the function on that input.

The OBDD is called reduced if it does not contain any vertex $v$ such that the 0 -edge and the 1-edge of $v$ lead to the same node, and it does not contain any distinct vertices $v$ and $v^{\prime}$ such that the subgraphs rooted in $v$ and $v^{\prime}$ are isomorphic. It is well-known that reduced OBDDs are a unique representation of switching functions $f: \mathbb{B}^{n} \rightarrow \mathbb{B}$ with respect to a given variable order [2]. The size of an OBDD is the number of its nodes. Several functions can be represented by a multi-rooted graph called shared $O B D D$. In the following, all functions are represented by a shared OBDD. Furthermore, typical OBDDimplementations use an additional edge attribute to represent a function and its complement by the same subgraph.

\subsection{Linear Transformations}

A Boolean function $\sigma: \mathbb{B}^{n} \rightarrow \mathbb{B}^{n}$ is called linear if there exists an $n \times n$-matrix $m_{\sigma}$ with Boolean entries such that $\sigma(x)=m_{\sigma}$. $x$ where the matrix product $m_{\sigma} \cdot x$ is evaluated over the Galois 
field $G F(2)$. Of course, $\sigma$ and $m_{\sigma}$ can be used interchangeably. Additionally we allow to complement some bits of $\sigma(x)$.

\section{Synthesis by Spectral Analysis}

In this section we revisit the algorithm of [3]. This description will form the basis for the development and judgement of our algorithm.

The problem: Given a Boolean function $f: \mathbb{B}^{n} \rightarrow \mathbb{B}$, find a linear transformation $\sigma$ and a remaining function $f^{\prime}$ : $\mathbb{B}^{n} \rightarrow \mathbb{B}$ such that the decomposition $f(x)=f^{\prime}(\sigma(x))$ simplifies the synthesis task and leads to smaller circuits.

The choice of suitable linear transformations is achieved by assigning each Boolean function a complexity measure that is heuristically related to the complexity of the final circuit implementation. The cost of the transformation $\sigma$ is neglected in this complexity measure on the grounds that the transformation itself is typically much less complex than the remaining function after the transformation. (In our experience this is true in most cases.)

In order to estimate the complexity of the Boolean function $f$ one can use the number of vector pairs $\left(x_{1}, x_{2}\right)$ which have a Hamming distance $d_{H}\left(x_{1}, x_{2}\right)$ of 1 and which have identical function values, i.e.

$$
C^{n}(f)=\#\left\{\left(x_{1}, x_{2}\right): d_{H}\left(x_{1}, x_{2}\right)=1 \text { and } f\left(x_{1}\right)=f\left(x_{2}\right)\right\} .
$$

This value lies in the range 0 to $n 2^{n}$. As a heuristic criterion, large values of $C^{n}(f)$ indicate a simple AND/ORimplementation with the extreme case $C^{n}(1)=n 2^{n}$. On the other hand, small values of $C^{n}(f)$ indicate an expensive AND/OR-implementation with the extreme case $C^{n}\left(x_{1} \oplus x_{2} \oplus\right.$ $\left.\cdots \oplus x_{n}\right)=0$. Due to the observation that many multilevel synthesis systems perform poorly without a good sumof-product representation of the target function, the authors of [3] claim that the complexity measure $C$ can also be used for multi-level synthesis.

It has been shown that the complexity measure $C(f)$ can be expressed by the Walsh spectrum of $f$ [4]. The Walsh spectrum of $f$ is the $2^{n}$ element vector

$$
S^{n}(f)=\left(s_{0}, \ldots, s_{2^{n}-1}\right)=W^{n} Y^{n}(f),
$$

where $Y^{n}(f)$ is the $2^{n}$ element truth table vector of $f$ (with $0,1$ replaced by $+1,-1)$ and $W^{n}$ is the $2^{n} \times 2^{n}$ Hadamard matrix defined by

$$
W^{n}=\left[\begin{array}{cc}
W^{n-1} & W^{n-1} \\
W^{n-1} & -W^{n-1}
\end{array}\right], \quad W^{0}=1 .
$$

If $\|u\|$ is defined as the number of 1 's in the binary representation of $u$, then the complexity measure $C$ can be written as a weighted sum of squares of the Walsh spectral coefficients

$$
C^{n}(f)=n 2^{n}-\frac{1}{2^{n}} \sum_{u=0}^{2^{n}-1}\|u\| s_{u}^{2}
$$

The main idea in [3] to find a suitable linear filter is to construct linear transformations that minimize the complexity $C$. In order to find suitable candidates for this minimization process the Walsh coefficient representation of the complexity measure is used. To avoid the exponential costs of computing the Walsh transformation symbolic BDD-techniques are used in connection with extensions of the Walsh transformation.

\section{Synthesis by Linear Sifting}

\subsection{The Linear Sifting Algorithm}

Originally starting from re-encodings of finite state machines and from the general optimization of BDD-representations two recent papers $[7,6]$ have investigated the effect of linear transformations on BDDs, concluding that they form a class very well suited to the implementation of efficient algorithms. In [6] an efficient algorithm is presented which combines the efficiency of Sifting and the power of linear transformations. We will briefly review this algorithm.

The well-known Sifting algorithm [12] tries to find a good variable ordering of an OBDD by successively investigating each variable: The current variable is moved through the whole ordering and finally put in the best position that has been found. The basic step during the movement of the variable is a pairwise exchange of variables. Suppose that variables $x_{i}$ and $x_{j}$ are to be swapped, and that $x_{i}$ immediately precedes $x_{j}$ before the swap. Then the effect of the exchange on each node labeled $x_{i}$ can be easily seen by applying Boole's expansion theorem w.r.t. both $x_{i}$ and $x_{j}$. Assuming $f$ is the function of a node labeled $x_{i}$, we have:

$$
f=x_{i} x_{j} f_{11}+x_{i} x_{j}^{\prime} f_{10}+x_{i}^{\prime} x_{j} f_{01}+x_{i}^{\prime} x_{j}^{\prime} f_{00} .
$$

Formally exchanging $x_{i}$ and $x_{j}$ and rearranging terms yields the function $\phi(f)$ :

$$
\phi(f)=x_{i} x_{j} f_{11}+x_{i} x_{j}^{\prime} f_{01}+x_{i}^{\prime} x_{j} f_{10}+x_{i}^{\prime} x_{j}^{\prime} f_{00} .
$$

In words, the effect of a swap is to interchange $f_{10}$ and $f_{01}$. If we repeat the same process for the application of an elementary linear transformation $x_{i} \mapsto x_{i} \equiv x_{j}$, we obtain:

$$
\phi(f)=x_{i} x_{j} f_{11}+x_{i} x_{j}^{\prime} f_{00}+x_{i}^{\prime} x_{j} f_{01}+x_{i}^{\prime} x_{j}^{\prime} f_{10} .
$$

A comparison of Equations (2)-(3) shows that the only difference is that $f_{00}$ is involved in the interchange with $f_{10}$, instead of $f_{01}$. The fundamental techniques applied to the efficient swapping of two variables can be used also for their linear combination. By combining swapping and linear transformations it is possible to reduce the size of the BDD in more cases than by swapping only. This observation forms the basis of the Linear Sifting algorithm, which proceeds as follows.

Each variable is considered in turn, and, as in Sifting, it is moved up and down in the order. Let $x_{i}$ be the chosen variable, and let $x_{j}$ be the variable immediately following it in the order. One basic step of Linear Sifting consists of the following three phases:

1. Variables $x_{i}$ and $x_{j}$ are swapped; let the size of the BDD after the swap be $s_{1}$.

2. The linear transformation $x_{j} \mapsto x_{i} \equiv x_{j}$ is applied; let the resulting size of the BDD be $s_{2}$.

3. If $s_{1} \leq s_{2}$ then the linear transformation is undone. This is obtained by simply applying the transformation again, since it is its own inverse.

The net effect of the three-phase procedure is that $x_{i}$ is moved one position onward in the order, and possibly linearly combined with $x_{j}$.

The usual formulation of linear transformations is in terms of exclusive or, rather than its complement. We use the equivalence function ( $\equiv$ ) because typically, BDDs use the complement arcs; hence, in order to preserve canonicity, we cannot swap $f_{11}$ with one of $\left\{f_{10}, f_{01}, f_{00}\right\}$, all of which could be complemented. Since the 1-arcs cannot be complemented, we would have to change the complementation of arcs into the 
node labeled (initially) $x_{i}$. This would make the operation non-local, which is highly undesirable.

The Linear Sifting algorithm is very time-efficient, as it is shown in [6], and it sometimes leads to significant reductions in the sizes of the OBDDs compared to the "conventional" Sifting algorithm.

\subsection{Applying Linear Sifting}

Our heuristic for extracting a linear filter is to use the (shared) BDD sizes of the functions $f^{\prime}, \sigma$ in the decomposition $f(x)=$ $f^{\prime}(\sigma(x))$ as a complexity measure:

$$
C^{n}\left(f^{\prime}, \sigma\right)=\operatorname{BDD}-\operatorname{size}\left(f^{\prime}, \sigma\right) .
$$

Our algorithm for extracting a linear filter is to apply the Linear Sifting algorithm on the given target function as explained above. Hence, our complexity measure coincides with the optimization criterion of the Linear Sifting algorithm. Particularly, we can exploit the fact that our implementation represents the linear transformation $\sigma$ within the shared BDD itself. The measure has been inspired by the observation that in some cases Linear Sifting yields significant reductions in the BDD size compared to the conventional Sifting algorithm. Although there is by far no strict correlation between BDD size and the difficulty in the mapping step, we expect functions with relatively small BDD size to be mapped easily. The advantage of our measure is that it is the most attractive one for the preprocessing step (minimizing the BDD size is equivalent to minimizing the memory consumption) and that it somehow seems to be particularly adequate to analyze Boolean functions whose implementation will be obtained through modern BDD-based logic synthesis tools.

Although we did not particularly aim at fully symbolic synthesis algorithms like the one by Minato [10] we also investigated these methods, see Section 5.3. These algorithms start by transforming the OBDD of a function into a ZDD (zerosuppressed BDD, [8]) of its prime-irredundant two-level form before proceeding.

\section{$5 \quad$ Experimental Results}

\subsection{Main Setup}

For the experimental evaluation we tried to use a very similar setup as the one in [3]. Our algorithm has been implemented using the CUDD BDD-package [14] and SIS 1.3 [13]. After building the BDDs in a suitable manner (see below) they are dumped to a BLIF file. In case of using Linear Sifting the transformation is also encoded into this file (in form of a subcircuit). Notice that, for sequential benchmarks, only the combinational portion of the logic has been considered, that is, state inputs and state outputs have been treated as primary inputs and primary outputs, respectively.

The functions are then synthesized under SIS 1.3 using script.rugged. Mapping was done using the library lib2.genlib with the command map -m 0 which optimizes the circuit for area. The memory limit was $128 \mathrm{MB}$, the time limit 20000 s on a Sun Sparc 10.

Our results for a set of 62 benchmark circuits are shown in Table 2. The column In represents the number of inputs resp. outputs of the function. The column Area shows the area of the mapped circuit, where all numbers are divided by 464 , the greatest common divisor of all gate sizes. The delay of the mapped circuit and the time that SIS needs for all the above mentioned tasks are listed in the next two columns. The

\begin{tabular}{|c|r||r|r|r||r|r|r|}
\hline & \multicolumn{1}{|c||}{} & \multicolumn{2}{c||}{ Starting from a HDL } & \multicolumn{3}{c|}{ Using transformation } \\
\hline & In & Area & Delay & BDD & Area & Delay & BDD \\
\hline \hline sec & 8 & 674 & 23.02 & 109 & 357 & 13.95 & 111 \\
\hline des & 256 & 6213 & 126.36 & 7388 & 7824 & 117.53 & 4887 \\
\hline
\end{tabular}

Figure 3: Reference results from [3].

following two columns show the size of the BDD after the preprocessing step ( $=$ the symbolic simulation using Sifting resp. Linear Sifting) and the running times of this preprocessing.

In our experiments we were especially interested in the question if it is possible by using an appropriate preprocessing to synthesize those functions which failed when using only conventional variable reordering. Hence, our experiments were conducted as follows: We used the publicly available very good variable orderings from the CUDD distribution [14] and used the mapping from the corresponding BDDs as our reference. These variable orderings were obtained by a large number of different variable reordering variants. Then we used three combinations of Sifting and Linear Sifting in order to see whether a circuit can be improved or a failed attempt can now be completed. The three combinations are:

1. Start from the well-known good ordering, then apply a final Linear Sifting.

2. Dynamically apply Linear Sifting.

3. Dynamically apply Linear Sifting until Convergence.

By these methods the number of fails could be reduced from 9 to 6 . As 2 of these 6 functions completed when starting from a good order, we now only have 4 functions that did not complete for any variant. Concerning the size of the circuits for which both approaches succeed, the decomposition generally seems to produce some overhead. However, in some cases like s1423, C7552 or C2670 significant gains could be achieved by using Linear Sifting. As it is proven by the CPU times, the preprocessing step is very time-efficient.

As a further reference we want to mention the results of [3]. Unfortunately, although they report on a large number of intermediate results of their implementation, they give their final mapping results only for two circuits whose BDDs have more than 31 nodes and compare it to a mapping from a hardware description language, see Fig. 3.

\section{$5.2 \quad$ Separate Mapping}

In the above described experimental setup, we were mainly interested in the effect of Linear Sifting when acting as a preprocessing for introducing some structure into the function representation.

In particular for the cases where Linear Sifting leads to far superior results we were interested in the question in how far it is advisable to encode the transformation and the remaining function into one BLIF file or to do the mappings separately. Table 4 shows what happens to the circuits C499, C1355, C1908 when mapping the transformation and the remaining function separately. It reflects the general observation that separate mapping typically does not improve the mapping results in our environment. However, we did not use a specialized algorithm for mapping the transformation (like in [3]). This might improve our results.

\subsection{Symbolic Synthesis}

Minato [10] has proposed the use of symbolic BDD- and ZDD techniques for optimizing multi-level logic circuits. They are 


\begin{tabular}{|c|c|c|c|c|c|c|c|c|c|c|c|}
\hline & & \multicolumn{5}{|c|}{ Starting from very good order } & \multicolumn{5}{|c|}{ Best results - three Linear Sifting BDDs } \\
\hline & In & Area & Delay & Time & BDD & PTime & Area & Delay & Time & BDD & PTime \\
\hline 9symml & $\overline{9}$ & 116 & 111.8 & 8 & 25 & 0.7 & 115 & 9.9 & 5 & 25 & 0.1 \\
\hline alu4 & 14 & 1210 & 36.8 & 156 & 350 & 0.2 & 1251 & 35.6 & 251 & 350 & 0.4 \\
\hline $\mathrm{t} 481$ & 16 & 90 & 12.5 & 3 & 21 & 0.9 & 90 & 12.5 & 4 & 21 & 1.0 \\
\hline $\mathrm{vda}$ & 17 & 1748 & 25.1 & 265 & 478 & 0.3 & 1800 & 29.7 & 269 & 478 & 0.5 \\
\hline cordic & 23 & 137 & 14.4 & 6 & 42 & 0.1 & 145 & 18.0 & 5 & 31 & 0.2 \\
\hline s499 & 23 & 561 & 19.8 & 49 & 330 & 0.1 & 1031 & 29.5 & 105 & 336 & 0.6 \\
\hline s344 & 24 & 300 & 18.2 & 12 & 104 & 0.1 & 366 & 18.3 & 14 & 83 & 0.3 \\
\hline ttt2 & 24 & 341 & 19.1 & 14 & 107 & 0.1 & 349 & 21.6 & 12.5 & 107 & 0.2 \\
\hline s1196 & 32 & 1509 & 29.3 & 362 & 598 & 0.3 & 1462 & 33.5 & 842 & 599 & 0.8 \\
\hline C1908 & 33 & \multicolumn{3}{|c|}{$\frac{1}{\text { TIME OUT }}$} & 5526 & 1.7 & 5148 & 74.9 & 3714 & 1209 & 23.1 \\
\hline adder 16 & 33 & 366 & 73.2 & 9 & 82 & 0.1 & 481 & 51.2 & 18 & 49 & 0.6 \\
\hline s635 & 34 & 569 & 40.4 & 24 & 128 & 0.2 & 569 & 40.4 & 25 & 128 & 0.6 \\
\hline $\mathrm{C} 432$ & 36 & 2308 & 43.5 & 1995 & 1064 & 0.3 & 2279 & 45.5 & 3168 & 1064 & 1.1 \\
\hline $\mathrm{mm} 9 \mathrm{~b}$ & 38 & 4472 & 46.9 & 6800 & 1527 & 0.7 & 4394 & 48.6 & 12941 & 1502 & 1.8 \\
\hline toolarge & 38 & 754 & 26.7 & 121 & 319 & 1.8 & 737 & 29.1 & 98 & 319 & 2.4 \\
\hline mm9a & 39 & 3851 & 52.5 & 4259 & 1111 & 0.5 & \multicolumn{3}{|c|}{ TIME OUT } & 980 & 2.0 \\
\hline C1355 & 41 & \multirow{2}{*}{\multicolumn{3}{|c|}{$\begin{array}{l}\text { TIME OUT } \\
\text { TIME OUT }\end{array}$}} & 25866 & 5.1 & 3110 & 54.6 & 757 & 484 & 18.1 \\
\hline $\begin{array}{l}\mathrm{C} 499 \\
\end{array}$ & 41 & & & & 25866 & 4.1 & 3419 & 58.0 & 968 & 520 & 10.7 \\
\hline k2 & 45 & 3667 & 35.2 & 1209 & 1246 & 0.6 & 3681 & 53.9 & 1438 & 1236 & 4.8 \\
\hline s967 & 45 & 1088 & 22.3 & 163 & 366 & 0.2 & 1012 & 24.3 & 169 & 383 & 0.5 \\
\hline C3540 & 50 & \multicolumn{3}{|c|}{ TIME OUT } & 23828 & 7.7 & \multicolumn{3}{|c|}{ TIME OUT } & 23823 & 120.2 \\
\hline s1269 & 55 & 3872 & 42.3 & \begin{tabular}{|l|}
16456 \\
\end{tabular} & 1695 & 0.4 & 3719 & 46.9 & 3573 & 1480 & 2.2 \\
\hline $\mathrm{C} 880$ & 60 & 13047 & 77.6 & 1761 & 4053 & 0.8 & 13150 & 78.7 & 1585 & 4052 & 5.2 \\
\hline comp32 & 64 & 568 & 80.1 & 42 & 97 & 0.1 & 527 & 76.2 & 38 & 97 & 0.7 \\
\hline adder32 & 65 & 734 & 146.2 & 30 & 162 & 0.2 & 1019 & 126.7 & 58 & 97 & 2.2 \\
\hline adsb32 & 65 & 1323 & 145.6 & 136 & 345 & 0.2 & 1323 & 110.6 & 82 & 191 & 1.5 \\
\hline s938 & 66 & 717 & 40.1 & 48 & 161 & 0.2 & 726 & 44.1 & 46 & 161 & 1.4 \\
\hline sbc & 68 & 1869 & 21.7 & 222 & 917 & 0.5 & 1861 & 19.6 & 193 & 917 & 2.0 \\
\hline alu32 & 73 & 2032 & 95.4 & 307 & 478 & 0.4 & 2078 & 82.1 & 281 & 388 & 1.8 \\
\hline alu32r & 73 & 2032 & 95.4 & 305 & 478 & 0.4 & 2100 & 95.1 & 279 & 388 & 3.0 \\
\hline dalu & 75 & 1665 & 32.5 & 488 & 689 & 0.8 & 1634 & 42.1 & 382 & 594 & 12.1 \\
\hline s991 & 84 & 1329 & 79.0 & 82 & 328 & 0.3 & 1485 & 116.6 & 82 & 328 & 1.8 \\
\hline $\mathrm{s} 1512$ & 86 & 1451 & 23.6 & 104 & 566 & 0.4 & 1358 & 29.5 & 102 & 526 & 1.6 \\
\hline xi30 & 90 & 675 & 157.4 & 22 & 91 & 0.3 & 675 & 157.4 & 20 & 91 & 1.3 \\
\hline s1423 & 91 & 3025 & 39.7 & 898 & 1796 & 0.6 & 2533 & 35.8 & 577 & 1477 & 11.4 \\
\hline dpath32 & 93 & 1776 & 133.6 & 272 & 485 & 0.4 & 1511 & 100.9 & 254 & 396 & 3.2 \\
\hline mm30a & 123 & \multicolumn{3}{|c|}{ MEMORY OUT } & 11065 & 4.5 & 27038 & 226.1 & 8470 & 8979 & 47.8 \\
\hline i3 & 132 & 254 & 9.9 & 11 & 133 & 0.2 & 290 & 11.0 & 13 & 133 & 1.0 \\
\hline i8 & 133 & 2788 & 45.4 & 541 & 1276 & 1.3 & 2860 & 52.9 & 719 & 1276 & 4.1 \\
\hline apex6 & 135 & 1406 & 25.2 & 84 & 498 & 0.3 & 1432 & 34.3 & 158 & 498 & 1.6 \\
\hline s3271 & 142 & 2564 & 29.0 & 160 & 831 & 0.8 & 2666 & 29.4 & 145 & 671 & 2.9 \\
\hline frg2 & 143 & 1823 & 36.1 & 313 & 963 & 0.6 & 1837 & 35.1 & 321 & 921 & 3.5 \\
\hline s4863 & 153 & \multicolumn{3}{|c|}{ MEMORY OUT } & 64088 & 26.3 & \multicolumn{3}{|c|}{ MEMORY OUT } & 64079 & 419.0 \\
\hline C5315 & 178 & 6374 & 53.0 & 542 & 1719 & 1.3 & 5746 & 64.1 & 3448 & 1537 & 8.5 \\
\hline s5378 & 199 & 3727 & 27.6 & 468 & 1932 & 1.3 & \multicolumn{3}{|c|}{ MEMORY OUT } & 1688 & 56.0 \\
\hline $\mathrm{C} 7552$ & 207 & 9189 & 143.3 & 638 & 2212 & 2.0 & 6182 & 113.5 & 315 & 1150 & 157.8 \\
\hline s3384 & 226 & 2605 & 83.3 & 141 & 693 & 1.2 & 2737 & 42.9 & 160 & 597 & 4.5 \\
\hline $\mathrm{C} 2670$ & 233 & 6475 & 71.1 & 433 & 1774 & 0.9 & 5003 & 51.8 & 308 & 1401 & 9.3 \\
\hline s9234.1 & 247 & 9007 & 45.1 & 733 & 3045 & 2.2 & 9045 & 48.1 & 688 & 2731 & 56.2 \\
\hline comp128 & 256 & 2296 & 318.9 & 3392 & 385 & 0.3 & 2103 & 311.2 & 2852 & 385 & 8.9 \\
\hline des & 256 & 7148 & 120.6 & 1950 & 2945 & 1.7 & 8106 & 224.9 & 4012 & 2087 & 162.5 \\
\hline $\operatorname{add} 128$ & 257 & 2942 & 584.5 & 1220 & 642 & 0.9 & 4091 & 497.3 & 189 & 385 & 46.2 \\
\hline adsb128 & 257 & 5435 & 579.5 & 10750 & 1401 & 1.4 & 5550 & 428.8 & 366 & 767 & 24.5 \\
\hline i10 & 257 & & $\overline{\mathrm{ME} \mathrm{Ol}}$ & & 20660 & 6.2 & & $\overline{\mathrm{ME} \mathrm{OL}}$ & & 19343 & 650.4 \\
\hline alu128 & 265 & & $\overline{\mathrm{ME} \mathrm{OI}}$ & & 1918 & 4.2 & 8550 & 323.4 & 623 & 1540 & 60.1 \\
\hline s6669 & 322 & & $\overline{\mathrm{ME} \mathrm{Ol}}$ & & 18033 & 25.3 & & $\overline{\mathrm{ME} \mathrm{OL}}$ & & 17912 & 157.3 \\
\hline clma & 415 & 1481 & $\begin{array}{l}33.3 \\
\end{array}$ & 117 & 738 & 9.0 & 1501 & 29.7 & 128 & 718 & 13.0 \\
\hline clmb & 415 & 1388 & 28.4 & 134 & 690 & 9.0 & 1244 & 23.7 & 103 & 618 & 24.2 \\
\hline dsip & 452 & 5310 & 119.7 & 1192 & 3033 & 3.1 & 10595 & 155.8 & 3848 & 2703 & 31.1 \\
\hline bigkey & 486 & 5529 & 126.5 & 1173 & 1595 & 1.4 & 7817 & 144.2 & 1480 & 1406 & 22.7 \\
\hline s 15850.1 & 611 & 25154 & 111.8 & 4766 & 9712 & 15.8 & 31514 & 137.6 & 7727 & 10934 & 236.7 \\
\hline s13207.1 & 700 & 7487 & 55.0 & 1322 & 2863 & 12.8 & 8622 & 43.2 & 1187 & 2854 & 42.0 \\
\hline
\end{tabular}

Figure 2: Experimental results. 


\begin{tabular}{|l|r||r|r|r||r|r|r||r|r||}
\hline & \multicolumn{1}{|c||}{} & \multicolumn{2}{|c||}{ Mapping remaining function } & \multicolumn{2}{|c||}{ Mapping transformation } & & \\
\hline & In & Area & Delay & Time & Area & Delay & Time & BDD & PTime \\
\hline \hline C1355 & 41 & 1667 & 26.0 & 82 & 1817 & 68.6 & 75.9 & 484 & 18.1 \\
\hline C499 & 41 & 1663 & 25.2 & 93 & 2296 & 83.2 & 83.7 & 520 & 10.7 \\
\hline C1908 & 33 & 4400 & 42.6 & 1906 & 732 & 29.9 & 15.9 & 1209 & 32.1 \\
\hline
\end{tabular}

Figure 4: Results from separate mapping.

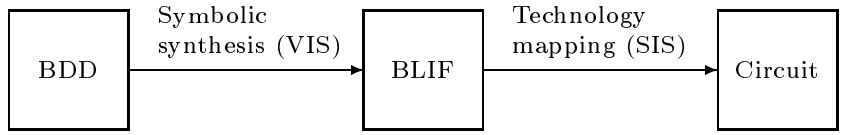

\begin{tabular}{|l|l||r|r|r|}
\hline & \multicolumn{1}{|c||}{} & \multicolumn{3}{c|}{ Mapping remaining function } \\
\hline & In & Area & Delay & Time \\
\hline \hline C1355 & 41 & 1945 & 22.5 & 314 \\
\hline C499 & 41 & 1801 & 28.9 & 191 \\
\hline C1908 & 33 & \multicolumn{3}{c|}{ TIME OUT } \\
\hline
\end{tabular}

Figure 5: Symbolic synthesis: Idea and results.

mainly based on generating a ZDD-representation for a primeirredundant two-level form of the given BDD and then using efficient divisor extraction algorithms on ZDDs for optimizing the circuit. As these techniques seem to be especially suited when starting a mapping procedure from a given BDD (instead e.g., of a multi-level description), we explored their use for our problem. In our experimental setup, we first used the symbolic algorithms for synthesizing the transformed function and then used SIS for doing the final mapping step, see Fig. 5. For the implementation of the symbolic synthesis algorithms we used the corresponding procedures of the newest VIS releases [1].

The results for some cases where Linear Sifting reduced the BDD size significantly are shown in Fig. 5. A comparison with Fig. 4 shows that the overhead from the two-phase mapping (VIS and SIS) seems to be too big in our context.

A very astonishing result which is also of independent interest in the connection between BDDs and ZDDs is obtained in the case of adders. When using Minato's algorithm to transform a BDD for an $n$-bit adder to the ZDD of its primeirredundant two-level form, the ZDD is of linear size. When applying Linear Sifting on the BDD of the adder, the BDD size is reduced by $40 \%$ of the original size. If, however, this linearly sifted BDD is transformed to the ZDD of its irredundant sum-of-product, the size of the resulting ZDD seems to grow exponentially. A thorough description of this effect can be found in the appendix.

\section{Conclusion}

We have presented and evaluated a new approach for synthesizing and mapping a Boolean function after performing a suitable decomposition. Our method is an alternative to the one proposed in [3]. The experimental results show that this approach may produce good results in cases where the function cannot be synthesized without a decomposition. Our approach is easy to implement in all environments which use dynamic reordering for BDDs, the resulting algorithm is simpler than the one in [3]. It also very well supports the concept of symbolic BDD-representations and may immediately profit from further improvements of the Linear Sifting algorithm.

In general, we think that the full power of decomposition techniques for mapping from a BDD has not been exploited so far. Additional investigations of the auxiliary routines (like
Linear Sifting), their combination and the optimization criteria should help to close this gap. Specifically, the results of the appendix suggest that the minimization of the ZDD for the cover of the given function rather than the minimization of the BDD for the function itself may provide a more reliable cost function. (The number of nodes in that ZDD corresponds to the number of literals in a factored form for the function.)

Consequently, we have begun the implementation of Linear Sifting for ZDDs and we expect results soon.

\section{REFERENCES}

[1] R. K. Brayton, G. D. Hachtel, A. Sangiovanni-Vincentelli, F. Somenzi, et al. VIS: A system $\mathrm{f}$. verification and synthesis. CAV '96, LNCS 1102, pp. 428-432. Springer, 1996.

[2] R. E. Bryant. Graph-based algorithms f. Boolean function manipulation. IEEE Trans. Comp., C-35:677-691, 1986.

[3] J. P. Hansen, M. Sekine. Synthesis by spectral translation using BDDs. In 33th DAC, pp. 248-253, 1996.

[4] S. L. Hurst, D. M. Miller, J. C. Muzio. Spectral Techniques in Digital Logic. Academic Press, 1985.

[5] Z. Kohavi. Switching and Finite Automata Theory. McGraw Hill, 1978.

[6] Ch. Meinel, F. Somenzi, T. Theobald. Linear sifting of decision diagrams. In 34th DAC, pp. 202-207, 1997.

[7] Ch. Meinel, T. Theobald. Local encoding transformations for optimizing OBDD-representations of FSMs. In FMCAD '96, LNCS 1166, pp. 404-418. Springer, 1996.

[8] S. Minato. Zero-suppressed BDDs for set manipulation in combinatorial problems. In 30th DAC, pp. 272-277, 1993.

[9] S. Minato. Binary Decision Diagrams and Applications for VLSI CAD. Kluwer Academic Publishers, 1996.

[10] S. Minato. Fast factorization method for implicit cube representation. IEEE Trans. CAD, 15:377-384, 1996.

[11] A. Narayan, J. Jain, M. Fujita, A. Sangiovanni-Vincentelli. Partioned OBDDs - a compact, canonical and efficiently manipulable representation for Boolean functions. In $I C C A D$ '96, pp. 547-553, 1996.

[12] R. Rudell. Dynamic variable ordering for ordered binary decision diagrams. In ICCAD '93, pp. 42-47, 1993.

[13] E. M. Sentovich, K. J. Singh, L. Lavagno, et al. SIS: A system for sequential circuit synthesis. Tech. Report UCB/ERL M92/41, Univ. of California, Berkeley, 1992.

[14] F. Somenzi. CUDD: Colorado University Decision Diagram Package. ftp://vlsi.colorado.edu/pub/, 1996.

[15] D. Varma, E. A. Trachtenberg. Design automation tools for efficient implementation of logic functions by decomposition. IEEE Trans. CAD, 8:901-916, 1989.

\section{Appendix}

In this appendix we give some analysis of adder functions. The goal is not only to analyze the blowup for adders reported in Section 5.3 but also to provide a new reference result for unexpected yet important effects caused by combining seemingly 


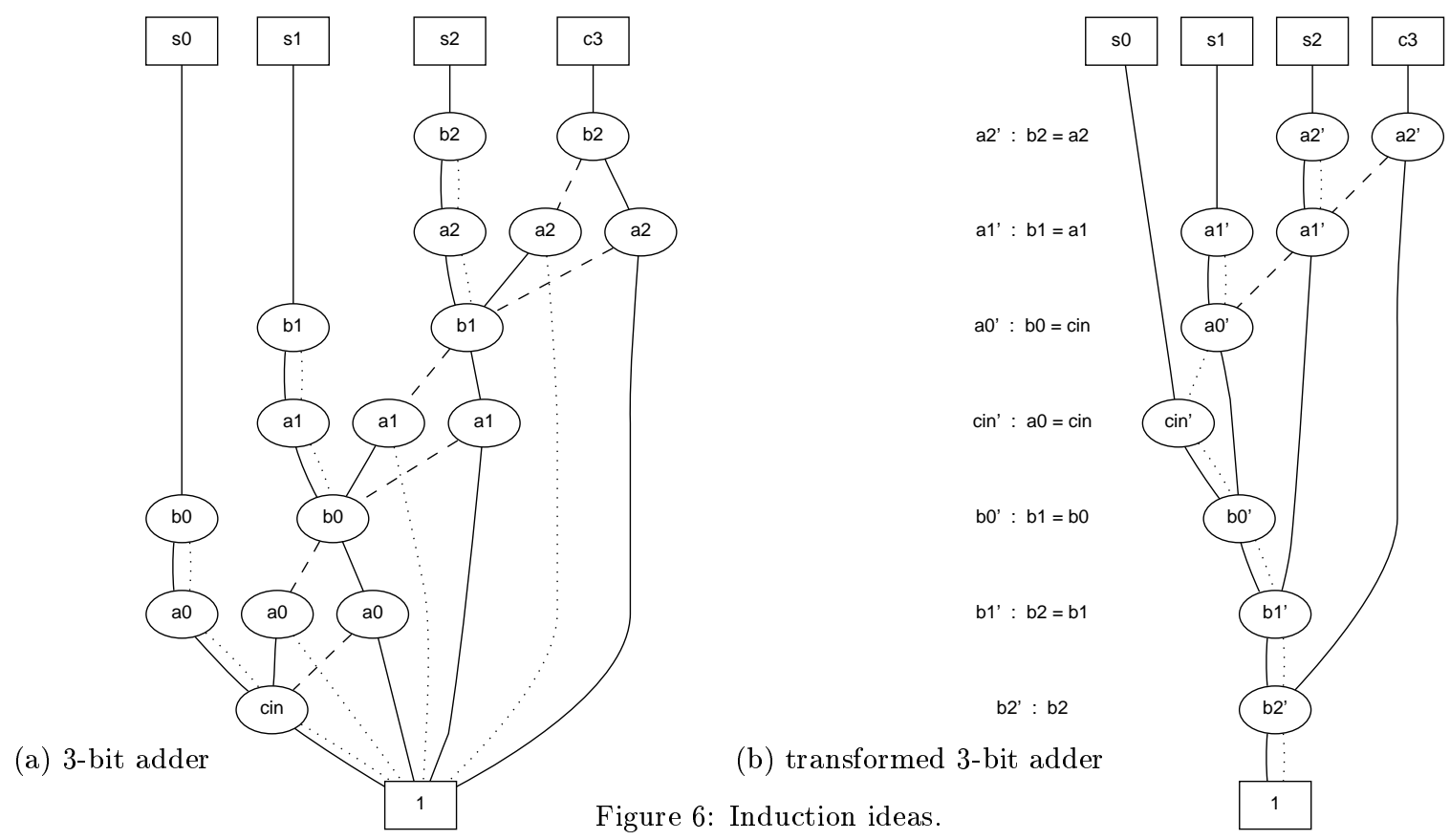

unrelated algorithms on BDDs and ZDDs.

Let $f\left(a_{n-1}, b_{n-1}, \ldots, a_{0}, b_{0}, c_{i n}\right): \mathbb{B}_{2 n+1} \rightarrow \mathbb{B}_{n+1}$ be the $n$-bit-adder function that takes as input two $n$-bit strings $a_{n-1} \ldots a_{0}, b_{n-1} \ldots b_{0}$ and an incoming carry $c_{i n}$, and computes their binary sum $s_{n-1} \ldots s_{0}$ and the outgoing carry bit $c_{n}$. It is well-known that good BDD-orders for the $n$-bit adder require to keep $a_{i}$ and $b_{i}$ together in the ordering, $0 \leq i \leq n-1$. Moreover, an optimal OBDD for the adder function that may use complemented edges needs at least $5 n+C$ nodes for a constant $C$. As it is shown in the following lemma the variable ordering $b_{n-1}, a_{n-1}, \ldots, b_{0}, a_{0}, c_{i n}$ leads to this optimal bound of (asymptotically) $5 n$.

Lemma 1 For $n>1$, the reduced $O B D D$ (using complemented edges) for the $n$-bit adder w.r.t. the variable ordering $b_{n-1}, a_{n-1}, \ldots, b_{0}, a_{0}, c_{i n}$ has exactly $5 n+2$ nodes.

Proof The lemma can be proven by induction over the number of variables. The idea of the induction should become clear from Fig. 6 (a) which shows the OBDD for a 3-bit adder. In the diagram a 1-edge is indicated by a solid line, a 0 -edge by a dashed line and a complemented 0 -edge by a dotted line. $\square$

Lemma 2 For $n \geq 1$, there exists a linear transformation $\rho_{n}$ such that the OBDD (using complemented edges) of the transformed $n$-bit adder has exactly $3 n+1$ nodes.

The transformation in the previous lemma is exactly the one that is found in our implementation of Linear Sifting.

Proof The proof is also by induction. The idea of the induction should become clear from Fig. 6 (b) which can be extended to arbitrary $n$. On the left side of the figure the functional relation between the original and the transformed variables is given.

The transformation from an OBDD to a ZDD for its primeirredundant sum-of-product is achieved by using Morreale's algorithm which has been adapted to the decision diagram environment by Minato [9]. As this ISOP algorithm contains a double recursion, a detailed analysis of the resulting ZDD function quickly becomes quite tedious. However, the following can be proved formally:
Lemma 3 For $n \geq 1$, the resulting $Z D D$ (which depends on $2 \cdot(2 n+1)$ variables) when applying Minato's algorithm on the adder of Lemma 1 has size $11 n+2$.

Proof Here, the inductive proof idea is that the ZDD for the $n$-bit adder has the structure which is shown in Fig. 7 with some subgraphs rooted in the nodes $A, B$. Each variable of the original OBDD is now represented by two variables representing the positive and the negative literal. The shown ZDD structure can be exploited recursively to construct the ZDD for the $(n+1)$-bit adder.

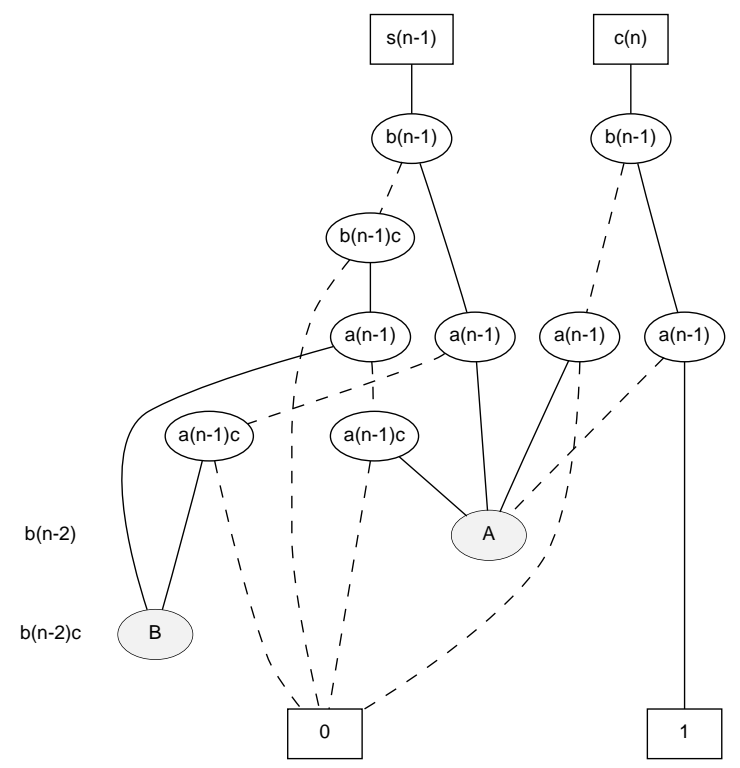

Figure 7: ZDD for the adder.

In contrast to the linear ZDD size resulting from the original adder, the ZDD size resulting from the transformed OBDD satisfies the recurrence equation size $(n)=2 \cdot \operatorname{size}(n-1)+4 n+5$ for all $n \geq 3$ in our corresponding experiments (in particular $n \leq 16)$ and hence seems to grow exponentially in $n$. 